\title{
Lapatinib inhibits the growth of esophageal squamous cell carcinoma and synergistically interacts with 5-fluorouracil in patient-derived xenograft models
}

\author{
WENMIN HOU ${ }^{1,5, *}$, XIA QIN ${ }^{2,3},^{*}$, XUEHUA ZHU ${ }^{6}$, MAOGUI FEI $^{1}$, PING LIU $^{2}$, LI LIU $^{3}$, \\ HANLIM MOON ${ }^{4}$, PINGKUAN ZHANG $^{4}$, JOEL GRESHOCK $^{3}$, KURTIS E. BACHMAN $^{3}$, \\ BANG-CE YE $^{5}$, HUI WANG ${ }^{1}$ and CRYSTAL YING QIN ZANG ${ }^{2,3}$
}

\begin{abstract}
${ }^{1}$ Key Laboratory of Nutrition and Metabolism, Institute for Nutritional Sciences,
Shanghai Institutes for Biological Sciences, Chinese Academy of Sciences, Graduate School of the Chinese Academy of Sciences, Shanghai 200031; ${ }^{2}$ Cancer Research, GlaxoSmithKline R\&D Center, Shanghai 201203, P.R. China;

${ }^{3}$ Cancer Research, GlaxoSmithKline, Collegeville, PA 19426, USA; ${ }^{4}$ Oncology Medicine, GlaxoSmithKline R\&D Center, Shanghai 201203; ${ }^{5}$ Laboratory of Biosystems and Microanalysis, State Key Laboratory of Bioreactor Engineering, East China University of Science and Technology, Shanghai 200237; ${ }^{6}$ Shanghai Key Laboratory of Signaling and

Disease Research, School of Life Science and Technology, Tongji University, Shanghai 200092, P.R. China
\end{abstract}

Received February 26, 2013; Accepted April 19, 2013

DOI: 10.3892/or.2013.2500

\begin{abstract}
Lapatinib is a dual tyrosine kinase inhibitor of epidermal growth factor receptor (EGFR) and human EGFR-2 (HER2) tyrosine kinase domains. To explore the potential utility of lapatinib for the treatment of esophageal squamous cell carcinoma (ESCC), we examined the expression profiles of EGFR and HER2 in tumor tissues and in paired adjacent non-neoplastic tissues from patients with ESCC. We evaluated the antitumor effects of lapatinib alone or in combination with oxaliplatin or 5-fluorouracil (5-FU) on a panel of primary ESCC cells in vitro with various levels of EGFR and HER2 expression. The in vivo effect of lapatinib alone or in combination with oxaliplatin or 5-FU was evaluated using a primary ESCC xenograft model. EGFR was overexpressed in $80.9 \%$ (76/94) of the ESCC samples, while 24.5\% (23/94) of the samples overexpressed HER2. EGFR and HER2 co-overexpression was detected in $22.3 \%$ of samples (21/94). In vitro, the primary ESCC cells were more sensitive to lapatinib combined
\end{abstract}

Correspondence to: Professor Hui Wang, Key Laboratory of Nutrition and Metabolism, Institute for Nutritional Sciences, Shanghai Institutes for Biological Sciences, Chinese Academy of Sciences, Shanghai 200031, P.R. China

E-mail: huiwang@sibs.ac.cn

Dr Crystal Ying Qin Zang, Cancer Research, GlaxoSmithKline R\&D Center, Shanghai 201203, P.R. China

E-mail: crystal.y.qin@gsk.com

*Contributed equally

Key words: lapatinib, esophageal squamous cell carcinoma, 5-fluorouracil, oxaliplatin with 5-FU or oxaliplatin than to lapatinib alone. Lapatinib in combination with 5-FU had more potent antitumor effects in the primary ESCC xenograft model, and markedly reduced the phosphorylation of EGFR and HER2, compared with lapatinib alone or in combination with oxaliplatin. These data indicate that lapatinib has activity in EGFR- and/or HER2-expressing ESCC primary cells, and that lapatinib in combination with 5-FU may be a promising treatment strategy for patients with ESCC.

\section{Introduction}

Although adenocarcinoma has replaced squamous cell carcinoma as the most common type of esophageal cancer in Western countries, $>90-95 \%$ of esophageal cancer cases in Asian countries are esophageal squamous cell carcinomas (ESCCs) (1-3). The prognosis of advanced ESCC patients in China and other Asian countries remains quite poor, despite the use of therapies that combine surgical resection with chemotherapy and/or radiotherapy. The use of tyrosine kinase inhibitors (TKIs) combined with standard chemotherapeutics may provide a novel therapeutic approach for the majority of ESCC patients (4-6).

The human epidermal growth factor receptor (EGFR) family, also known as the HER family, includes four closely related receptors: HER1 (EGFR), HER2, HER3 and HER4. Downstream signaling of the HER family plays a crucial role in cell proliferation, apoptosis, angiogenesis and metastasis $(7,8)$. EGFR overexpression has been identified in several types of human cancer, including gastric, colorectal, breast, lung, prostate and bladder cancer $(9,10)$. The HER2 gene is amplified and overexpressed in $\sim 30 \%$ of human breast and ovarian cancers, as well as other tumors, including colorectal and gastric cancer (11-13). Notably, it was previously reported 
that EGFR is overexpressed in $33.3 \%$ of Japanese ESCC patients, and HER2 is overexpressed in $30.3 \%$ of Japanese ESCC patients $(14,15)$. EGFR and HER2 are the main therapeutic targets of TKIs, and oxaliplatin and 5-FU are used as standard chemotherapies (16-18). Thus, anti-EGFR and/or anti-HER 2 targeted therapy combined with standard chemotherapies is an attractive approach for the treatment of patients with advanced ESCC.

Lapatinib is a reversible dual TKI that targets the tyrosine kinases in both EGFR and HER2 tyrosine kinases, which in turn inhibits receptor phosphorylation and activation of the downstream signaling pathways, such as extracellular-related kinase (ERK)-1/2 and AKT in cell lines and xenografts (19-21). Lapatinib combined with fluoropyrimidine or trastuzumab exerted synergistic antitumor effects in vitro and in vivo (22), and had clinical activities in several solid tumors $(6,23,24)$. However, no previous studies have described the effects of this TKI combined with standard chemotherapy using ESCC primary xenografts derived from Chinese patients. On the basis of these earlier observations, we explored the potential utility of lapatinib when administered alone or in combination with oxaliplatin and 5-FU for treating ESCC.

\section{Materials and methods}

Ethics statement. The present study was conducted according to the principles of the Declaration of Helsinki. The efficacy study was approved by the Ethics Committee of the Third Xiangya Hospital, Central South University, Hunan, China. The collection and use of the archived paraffin tissue blocks in the ESCC study were approved by the Ethics Committee of the Second Hospital, Jilin University, Jilin, China, with prior consent from the patients.

Immunohistochemical analysis. Esophageal cancer tissue microarrays (TMA; Shanghai Outdo, Shanghai, China) comprising 94 malignant esophageal human tumors in which 78 had matched adjacent normal tissues were used for immunohistochemistry (IHC) analysis of EGFR and HER2 protein expression. For antigen retrieval, the tissues were incubated in EDTA buffer with heating for $20 \mathrm{~min}$, treated with $3 \%$ hydrogen peroxide, and then incubated in Background Sniper (Biocare Medical, Concord, CA, USA) to block any non-specific binding. Sections were then incubated with the anti-EGFR or anti-HER 2 monoclonal antibody (Cell Signaling Technology, Beverly, MA, USA) diluted in Dako antibody diluent (DakoCytomation, Carpinteria, CA, USA) overnight at $4^{\circ} \mathrm{C}$. The sections were then incubated using the rabbit on rodent polymer system (Biocare Medical) for $30 \mathrm{~min}$ at room temperature. Slides were subsequently treated with 3,3-diaminobenzidine chromogen (DakoCytomation) for 5 min to visualize antibody binding. Sections were then counterstained with hematoxylin, dehydrated and mounted. Total rabbit $\mathrm{IgG}$ substituted for the primary antibodies served as a negative control.

The assessment of EGFR or HER2 staining strength and positivity was carried out simultaneously by a pathologist and two other observers and a consensus was reached for each core. EGFR and HER2 staining was scored as follows: negative, no membranous staining in any of the tumor cells;
$1+$, membranous staining in $<10 \%$ of the tumor cells with any intensity or in $<30 \%$ of the tumor cells with weak intensity; $2+$, staining in $10-30 \%$ of the tumor cells with moderate to strong intensity or staining in $30-50 \%$ of the tumor cells with weak to moderate intensity; and $3+$, staining in $>30 \%$ of the tumor cells with strong intensity or $>50 \%$ of the tumor cells with any intensity. Tissues scored as $2+$ or $3+$ were defined as showing positive expression (25).

Animals. Athymic nude mice (Vr: NU-Foxn1nu) aged 5-6 weeks and weighing 18-21 g were purchased from Vital River Laboratories (Beijing, China). The health of all mice was monitored daily by gross observation and analysis of blood samples of sentinel animals. All mice were allowed to acclimatize and recover from any shipping-related stress for $\geq 72 \mathrm{~h}$ prior to experimental use. Autoclaved water and irradiated food were provided, and the mice were maintained on a $12 \mathrm{~h}$ light and dark cycle. Cages, bedding and water bottles were autoclaved before use and were changed weekly.

All animal experiments were conducted in an Association for Assessment and Accreditation of Laboratory Animal Careaccredited animal facility after review by the Institutional Animal Care and Use Committees, and in accordance with the GSK policy on the Care, Welfare and Treatment of Laboratory Animals.

Tumors. Excess human esophageal tumor samples were obtained through an institutional review board-approved centralized banking infrastructure at the hospital. Written informed consent was obtained from all participants. None of the samples used in the present study were derived from minors. Biopsies were taken from the luminal surface of resected specimens by a pathologist or surgeon, ensuring that their potential for histopathologic diagnosis and staging was not compromised.

Solid tumor tissues were depleted of necrotic components, cut into 10-15 mg pieces and mixed. The mixed tumor pieces were implanted (single flank) into male $\mathrm{Nu} / \mathrm{Nu}$ nude mice; 3-5 pieces were mixed with 15-30 $\mu 1$ Matrigel (BD Biosciences, Bedford, MA, USA) per mouse. For continued propagation in mice, the xenograft tumors were excised and processed into mixed tumor pieces. The mixed tumor pieces were re-implanted subcutaneously into new recipient nude mice. All of the primary human esophageal tumors used in this study had undergone 3-4 passages in vivo, and the histologic profiles of all tumors were maintained during serial transplantation.

In vitro ATP tumor chemosensitivity assay. Chemosensitivity was assessed in primary esophageal tumor tissue samples using the CellTiter-Glo ${ }^{\circledR}$ Luminescent Cell Viability Assay kit (Promega, Madison, WI, USA). Briefly, surgical biopsies $\left(1-2 \mathrm{~cm}^{3}\right)$ were obtained during primary surgery. Tumor cells were isolated by mechanical and enzymatic dissociation. Approximately $2 \times 10^{4}$ cells were then seeded into each well of a 96-well polypropylene microplate. Each concentration of the test drugs was applied in triplicate. The initial concentrations of lapatinib, oxaliplatin and 5-FU were 10, 10 and $100 \mu \mathrm{M}$, respectively. Two rows on each plate were reserved for blanks and controls. After preparing the diluted drugs, $135 \mu \mathrm{l}$ of the cell suspension was added to each well. The plate was incu- 
A
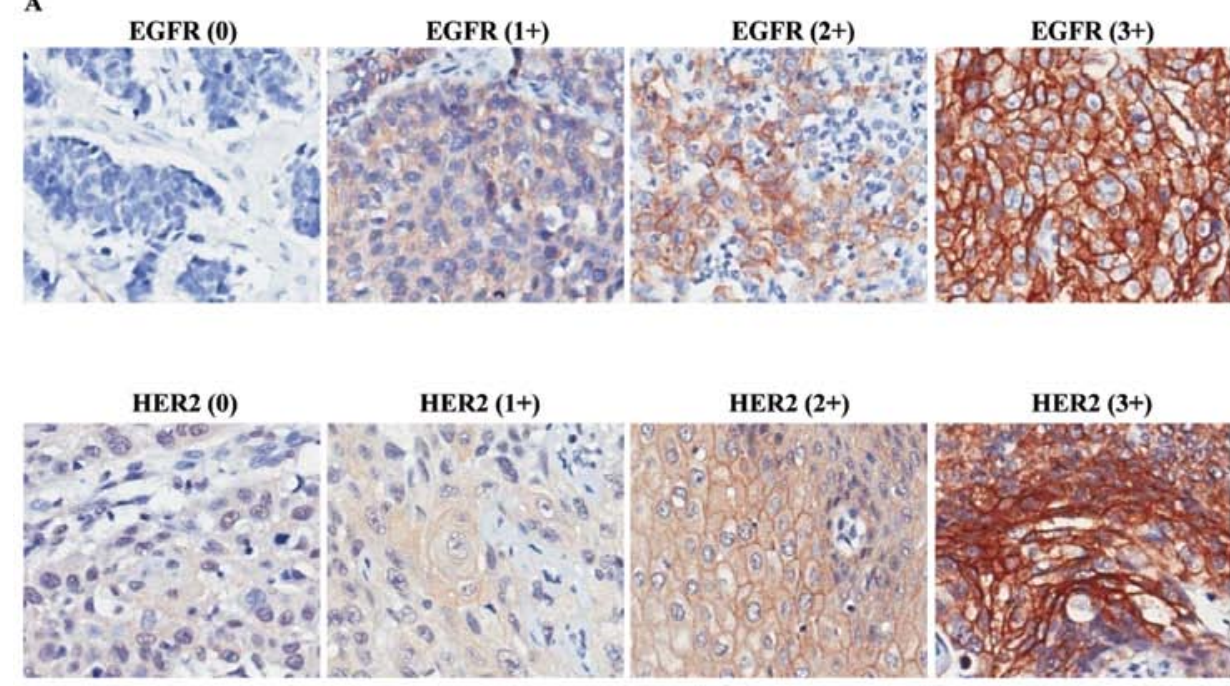
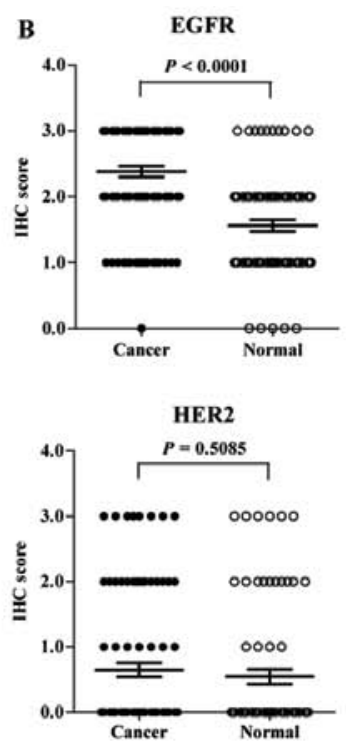

Figure 1. Immunohistochemical analysis of EGFR and HER2 in ESCC tissue samples from Chinese patients. EGFR and HER2 expression levels were evaluated by immunohistochemical staining of serial sections of 94 primary esophageal tumors. (A) Representative staining of EGFR and HER2 in ESCC tumor tissue. Original magnification, x200. (B) Scatter plot analysis of EGFR and HER2 membrane expression in ESCC tumor tissues and adjacent normal tissues.

bated for 6 days at $37^{\circ} \mathrm{C}$ under high humidity and $5 \% \mathrm{CO}_{2}$. The cells were observed microscopically every $24 \mathrm{~h}$ to check for overgrowth or infection. At the end of the incubation period, the cells were lysed by the addition of $75 \mu \mathrm{l}$ of CellTiter-Glo reagent. Luminescence measurements were made using a FlexStation 3 (Molecular Devices, Sunnyvale, CA, USA).

Immunoblotting. Tumor tissue lysates were prepared by washing the cells with phosphate-buffered saline and subjecting them to lysis with radio-immunoprecipitation assay buffer supplemented with a protease inhibitor cocktail. The protein concentrations were quantified using the Bio-Rad protein assay kit (Bio-Rad Laboratories, Hercules, CA, USA). Equivalent amounts of proteins were loaded, separated on NuPAGE Novex 4-12\% Bis-Tris Gels, and then transferred to polyvinyl difluoride membranes (Invitrogen, Carlsbad, CA, USA). The membranes were blocked for $1 \mathrm{~h}$ with $5 \%$ nonfat dried milk in Tris-buffer containing $0.1 \%$ Tween and were then probed with the diluted primary antibody overnight at $4^{\circ} \mathrm{C}$. The membranes were then washed three times and probed with horseradish peroxidase-linked goat anti-rabbit $\mathrm{IgG}$, and the immunoreactive bands were visualized using an enhanced chemiluminescent detection system (GE Healthcare, Piscataway, NJ, USA). All antibodies were purchased from Cell Signaling Technology.

Test agents and efficacy of study design. Lapatinib (Tykerb ${ }^{\circledR}$; GlaxoSmithKline, Research Triangle Park, NC, USA) was prepared in $0.5 \%$ hydroxypropyl methylcellulose (HPMC) and 0.1\% Tween-80 (Sigma, St. Louis, MO, USA). Clinical-grade oxaliplatin (Eloxatin ${ }^{\circledR}$; Sanofi-Aventis, Bridgewater, NJ, USA) was obtained as a stock solution of $5 \mathrm{mg} / \mathrm{ml}$, diluted with sterile water. 5-FU (Sigma) was freshly dissolved in saline prior to use.

Administration of lapatinib and oxaliplatin in the primary esophageal tumor model. Forty-two mice were selected and randomized into six groups. The mice were treated with vehicle (0.5\% HPMC in $0.1 \%$ Tween-80, orally, twice daily), lapatinib alone $(105 \mathrm{mg} / \mathrm{kg}$, orally, twice daily), oxaliplatin alone (6 mg/kg, intraperitoneal injection, once weekly), or lapatinib (105 mg/kg, orally, twice daily) in combination with oxaliplatin (6 mg/kg, intraperitoneal injection, once weekly) for 3 weeks.

Administration of lapatinib and 5-FU in the primary esophageal tumor model. Forty two mice were selected and randomized into six groups. The mice were treated with vehicle (0.5\% HPMC in $0.1 \%$ Tween-80, orally, twice daily), lapatinib alone (105 mg/kg, orally, twice daily), 5-FU alone $(15 \mathrm{mg} / \mathrm{kg}$, intraperitoneal injection, once daily for 4 days per week), or lapatinib (105 mg/kg, orally, twice daily) in combination with 5 -FU $(15 \mathrm{mg} / \mathrm{kg}$, intraperitoneal injection, once daily for 4 days per week) for 3 weeks.

Measurement of tumor growth and body size. Tumor volume was calculated using the following formula: tumor volume $=\left(\right.$ length $\mathrm{x}$ width $\left.{ }^{2}\right) / 2$. The tumor volume was then used to calculate tumor growth inhibition (TGI), as an index of the antitumor activity of each test drug, as follows: TGI $(\%)=[1-$ $\left.\left(\mathrm{Ti}-\mathrm{T}_{0}\right) /\left(\mathrm{Vi}-\mathrm{V}_{0}\right)\right] \mathrm{x} 100$; where $\mathrm{Ti}$ is the mean tumor volume of the treated group, $\mathrm{T}_{0}$ is the mean tumor volume of the treated group on Day 1 of treatment, $\mathrm{Vi}$ is the mean tumor volume of the vehicle-treated group, and $\mathrm{V}_{0}$ is the mean tumor volume of the vehicle-treated group on Day 1 of treatment. Tumor weight inhibition (TWI) was calculated at the end of the study using the following formula: TWI $(\%)=\left(1-\mathrm{T}_{\mathrm{TW}} / \mathrm{V}_{\mathrm{TW}}\right) \times 100$; where $\mathrm{T}_{\mathrm{TW}}$ is the mean tumor weight of the treated group on the final day of the study and $\mathrm{V}_{\mathrm{TW}}$ is the mean tumor weight of the vehicle-treated group on the final day of the study. Efficacy data presented as the mean tumor volume \pm standard error of the mean (SEM).

The relative change in body weight $(\mathrm{RCBW})$ in each mouse was calculated using the following formula: RCBW 
$(\%)=\left(\mathrm{BW}_{\mathrm{i}}-\mathrm{BW}_{0}\right) / \mathrm{BW}_{0} \times 100$; where $\mathrm{BW}_{\mathrm{i}}$ is the mean body weight on Day $\mathrm{i}$ and $\mathrm{BW}_{0}$ is the mean body weight on Day 0 . The mean, standard deviation and SEM was calculated for each group.

Tolerability measures. Total body weight was used as a surrogate endpoint for tolerability in all studies.

Statistical analyses. Statistical analyses were carried out using GraphPad Prism 5 software (GraphPad Software Inc., San Diego, CA, USA). Student's t-test was used to compare EGFR and HER2 expression levels in tumor tissues with those in adjacent normal tissues. The survival curve was drawn by a Kaplan-Meier method, and the statistical significance was assessed by the Gehan-Breslow-Wilcoxon test. In efficacy studies, the treated groups were compared with the vehicle group using repeated-measures analysis of variance and Dunnett's post-hoc test. Differences between groups were considered statistically significant at $\mathrm{P}<0.05$, as very significant at $\mathrm{P}<0.01$ and as highly significant at $\mathrm{P}<0.001$.

\section{Results}

EGFR and HER2 overexpression in ESCCs from Chinese patients. Previous studies have highlighted the role of the EGFR signaling pathway in the development and progression of solid tumors (10). Therefore, we used IHC to examine the expression of EGFR and HER2 in serial sections of ESCC collected from 94 patients. As illustrated by the examples shown in Fig. 1A, the level and distribution of EGFR and HER2 expression varied widely among the analyzed tumors. EGFR expression was positive in 76 samples (80.9\%), while HER 2 expression was positive in 23 samples (24.5\%). Twenty-one samples (22.3\%) were positive for both EGFR and HER2. The scatter plots in Fig. 1B show the distribution of EGFR or HER2 IHC scores in ESCC tumor tissues as compared with those in adjacent normal tissues. EGFR expression was significantly higher in ESCC tumor tissues than in adjacent normal tissues $(\mathrm{P}<0.0001)$, whereas there was no significant difference in HER2 expression. The distribution of IHC staining of EGFR and HER2 indicated that there was no correlation between EGFR and HER2 expression in these ESCCs (Table I). Of note, the HER2-positive patients were more likely to be EGFR-positive than not, although the difference was not statistically significant. The survival rate of patients positive for both EGFR and HER2 tended to be higher than that of patients who were negative for both EGFR and HER2 (Fig. 2). Taken together, these results suggest that the EGFR and HER2 signaling pathway is activated in these ESCCs obtained from Chinese patients.

EGFR and HER2 expression in primary esophageal tumor models. We next examined the total protein expression levels and phosphorylation levels of EGFR and HER 2 in primary tumor cells derived from ESCCs from Chinese patients $(\mathrm{n}=7)$. The levels of unphosphorylated and phosphorylated EGFR and HER2 proteins were highly variable among the primary ESCC cells. As shown in Fig. 3A, five out of seven primary tumor cells (ESX001, ESX008, ESX009, ESX026 and ESX030) expressed high levels of EGFR. Five tumor cells
Table I. EGFR and HER2 expression pattern in ESCCs obtained from 94 Chinese patients.

\begin{tabular}{|c|c|c|c|c|c|}
\hline & \multicolumn{2}{|c|}{ HER2 (+) } & \multicolumn{2}{|c|}{ HER2 (-) } & \multirow[b]{2}{*}{ P-value } \\
\hline & No. & $\%$ & No. & $\%$ & \\
\hline EGFR (+) & 21 & 22.3 & 55 & 58.5 & 0.1093 \\
\hline EGFR (-) & 2 & 2.1 & 16 & 17 & \\
\hline
\end{tabular}

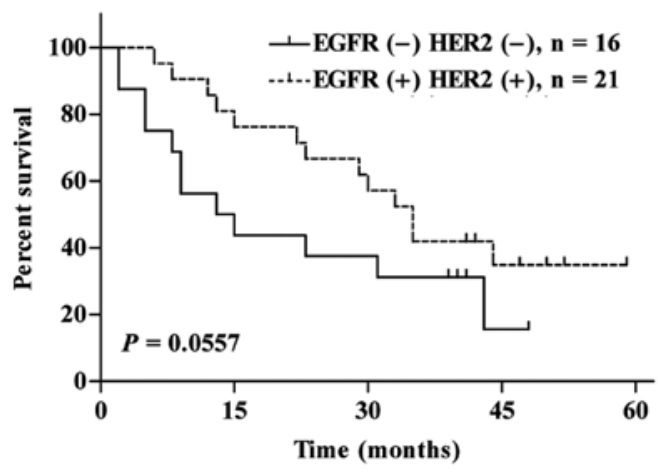

Figure 2. Survival curves of patients according to EGFR and HER2 expression The survival rates were analyzed by the Kaplan-Meier method, and survival was determined from the time of operation to mortality or the last follow-up.

(ESX002, ESX007, ESX008, ESX009 and ESX030), particularly ESX007, expressed high levels of total HER2 protein. The level of phosphorylated EGFR or HER2 expression also varied widely. Of the seven primary ESCC tumor cells, most of the cells expressed phosphorylated EGFR or HER2. Very high levels of phosphorylated EGFR (ESX008) or phosphorylated HER2 (ESX007) were observed in one cell line each. These data indicate that EGFR and HER2 are highly expressed and their signaling pathways are activated in ESCCs obtained from Chinese patients.

Inhibitory effects of lapatinib in combination with oxaliplatin or 5-FU on the growth of primary ESCC cells. It is well established that lapatinib inhibits protein phosphorylation in cells overexpressing HER2 (19-21). Therefore, we assessed the inhibitory effects of lapatinib, oxaliplatin, and 5-FU alone or lapatinib in combination with either oxaliplatin or 5-FU on the growth of seven primary ESCC cells. The cells were observed microscopically to assess morphologic changes after 7 days of treatment. In the seven primary ESCC cells, lapatinib and 5-FU both inhibited cell proliferation in concentration-dependent manners, with a calculated $\mathrm{IC}_{50}$ of 1-10 $\mu \mathrm{M}$. Oxaliplatin also inhibited proliferation with similar $\mathrm{IC}_{50}$ values for six of the seven primary cells; the exception was ESX030, which was resistant to oxaliplatin in vitro (Fig. 3B).

The effect of lapatinib in combination with standard chemotherapy was examined to determine the nature of the interaction (i.e., synergistic, additive or antagonistic). As compared with lapatinib in combination with oxaliplatin, lapatinib in combination with 5-FU showed some evidence of synergy in primary tumor cells, although this was not significant (Table II). 
Table II. Antitumor effects of lapatinib, oxaliplatin and 5-FU alone or lapatinib in combination with oxaliplatin or 5-FU in primary ESCC cells.

\begin{tabular}{|c|c|c|c|c|c|c|c|}
\hline \multirow{2}{*}{$\begin{array}{l}\text { ESCC } \\
\text { primary } \\
\text { cells }\end{array}$} & \multicolumn{3}{|c|}{$\begin{array}{l}\text { Single agent } \mathrm{IC}_{50} \\
(\text { mean } \pm \mathrm{SD}, \mu \mathrm{M})\end{array}$} & \multicolumn{2}{|c|}{$\begin{array}{l}\text { Combination } 1 \mathrm{IC}_{50} \\
(\text { mean } \pm \mathrm{SD}, \mu \mathrm{M})\end{array}$} & \multicolumn{2}{|c|}{$\begin{array}{l}\text { Combination } 2 \mathrm{IC}_{50} \\
(\text { mean } \pm \mathrm{SD}, \mu \mathrm{M})\end{array}$} \\
\hline & Lapatinib & Oxaliplatin & $5-\mathrm{FU}$ & Lapatinib & Oxaliplatin & Lapatinib & 5-FU \\
\hline ESX001 & $1.96 \pm 0.01$ & $2.29 \pm 0.43$ & $2.84 \pm 0.22$ & $1.01 \pm 0.13$ & $1.01 \pm 0.13$ & $0.33 \pm 0.02$ & $3.32 \pm 0.19$ \\
\hline ESX002 & $1.32 \pm 0.36$ & $4.59 \pm 0.39$ & $2.43 \pm 0.31$ & $0.95 \pm 0.08$ & $0.95 \pm 0.08$ & $0.24 \pm 0.03$ & $2.39 \pm 0.30$ \\
\hline ESX007 & $2.47 \pm 0.26$ & $2.82 \pm 0.22$ & $3.48 \pm 0.34$ & $1.28 \pm 0.08$ & $1.28 \pm 0.08$ & $0.41 \pm 0.03$ & $4.03 \pm 0.29$ \\
\hline ESX008 & $1.99 \pm 0.03$ & $5.70 \pm 0.41$ & $3.39 \pm 0.13$ & $1.29 \pm 0.09$ & $1.29 \pm 0.09$ & $0.29 \pm 0.03$ & $2.93 \pm 0.26$ \\
\hline ESX009 & $2.53 \pm 0.30$ & $3.61 \pm 0.30$ & $3.37 \pm 0.16$ & $1.99 \pm 0.29$ & $1.99 \pm 0.29$ & $0.35 \pm 0.00$ & $3.47 \pm 0.04$ \\
\hline ESX026 & $2.00 \pm 0.04$ & $5.49 \pm 1.32$ & $2.68 \pm 0.16$ & $1.51 \pm 0.20$ & $1.51 \pm 0.20$ & $0.32 \pm 0.02$ & $3.21 \pm 0.24$ \\
\hline ESX030 & $6.00 \pm 3.03$ & $>10$ & $7.92 \pm 1.28$ & $2.80 \pm 0.32$ & $2.80 \pm 0.32$ & $0.92 \pm 0.08$ & $9.18 \pm 0.85$ \\
\hline
\end{tabular}

A

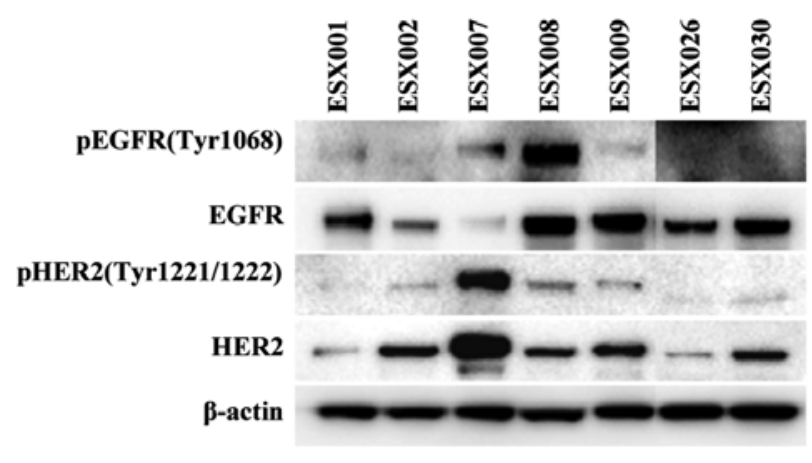

B

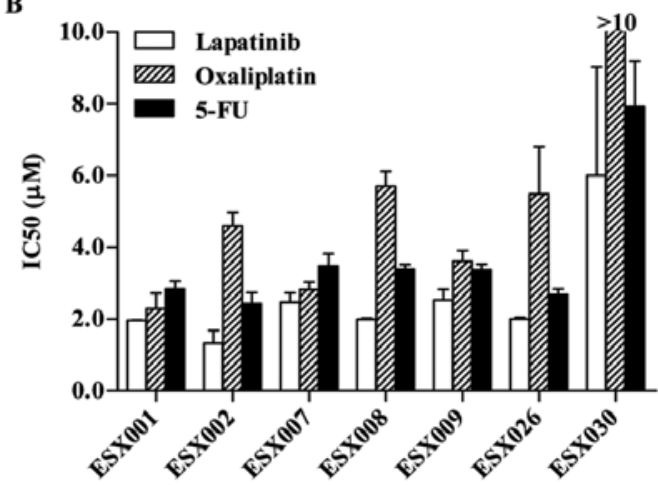

Figure 3. Protein expression profiles in ESCC models and in vitro responsiveness to lapatinib, oxaliplatin and 5-FU. (A) Seven primary ESCC tumors derived from cancer patients (ESX001, ESX002, ESX007, ESX008, ESX009, ESX026 and ESX030) were lysed and subjected to western blotting of the phosphorylated and unphosphorylated EGFR2 and HER2 protein. Data shown represent one of three independent experiments, each with comparable results. (B) Seven primary ESCC cells derived from cancer patients were treated with DMSO or increasing doses of lapatinib (0-10 $\mu$ M), oxaliplatin or 5-FU for 6 days. Following incubation, the tumor chemosensitivity assay was carried out. The data represent the results of triplicate experiments.

Effects of lapatinib, oxaliplatin and 5-FU on the primary ESCC model

Lapatinib in combination with oxaliplatin. After 21 days of treatment, lapatinib and oxaliplatin administered alone at doses of 105 and $6 \mathrm{mg} / \mathrm{kg}$, respectively, did not appear to inhibit tumor cell growth in the primary ESCC model. The TGIs for lapatinib and oxaliplatin alone were 29.65 and $26.22 \%$, respectively, and the TWIs were 21.95 and $22.88 \%$, respectively. However, administration of lapatinib in combination with oxaliplatin at the same doses had significantly greater inhibitory effects compared with vehicle $(\mathrm{P}<0.001$; Fig. 4A), with a TGI and TWI of 51.42 and $44.55 \%$, respectively (Fig. 4E and F).

Lapatinib in combination with 5-FU. Consistent with the above experiment, lapatinib administered at a dose of $105 \mathrm{mg} / \mathrm{kg}$ had no obvious antitumor effects in the ESCC primary model, with a TGI and TWI of 23.71 and $13.51 \%$, respectively. The antitumor effect of lapatinib alone was not significantly different from that of vehicle ( $\mathrm{P}>0.05)$. By contrast, 5-FU administered at $15 \mathrm{mg} / \mathrm{kg}$ four times per week moderately inhibited tumor growth; the TGI and TWI for 5-FU were 57.25 and $44.54 \%$, respectively (Fig. 4E and F). The antitumor effect of 5-FU alone was significantly greater than that of vehicle $(\mathrm{P}<0.05)$. Combining lapatinib with 5-FU at the same doses resulted in greater tumor growth inhibition compared with either drug alone (Fig. 4B). The antitumor effect of lapatinib in combination with 5-FU (TGI=94.05\%) was significantly greater than that of vehicle $(\mathrm{P}<0.001)$ and compared with lapatinib alone $(\mathrm{P}<0.05)$. The TWI for lapatinib in combination with 5-FU $(68.82 \%)$ was also greater than that of lapatinib or 5-FU alone.

Tolerability. There was no evidence for toxicities with lapatinib alone or in combination with oxaliplatin or 5-FU in athymic mice bearing human primary ESCC tumors. There were no marked changes in RCBW during the study, or any significant differences in RCBW between each treatment group (Fig. 4C and D).

Synergistic antitumor effects of lapatinib and 5-FU against ESCCs ex vivo. The phosphorylated and total protein levels of several downstream markers were measured in mice treated with a single agent or lapatinib in combination with oxaliplatin (Fig. 5A) or 5-FU (Fig. 5B). Lapatinib in combination with 
A

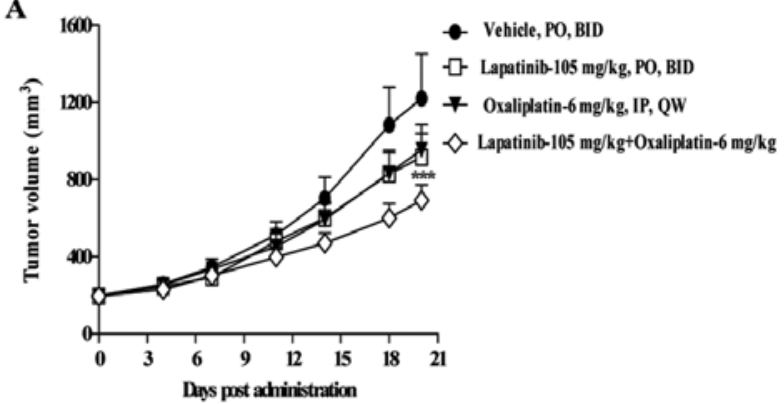

C

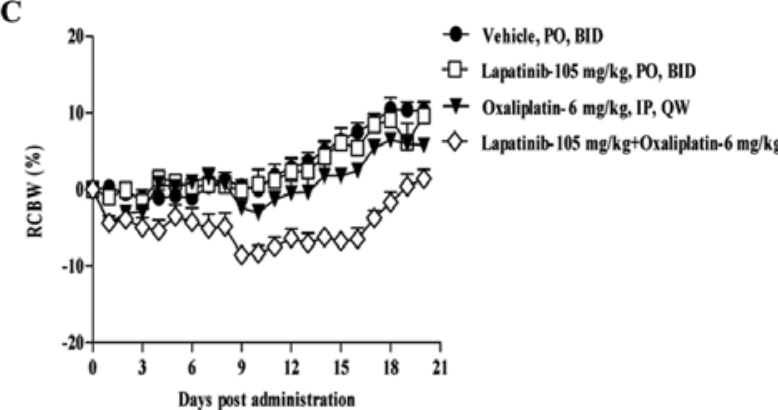

E
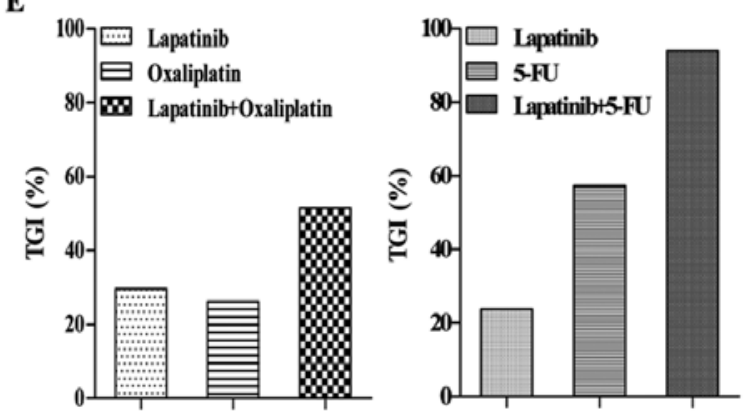

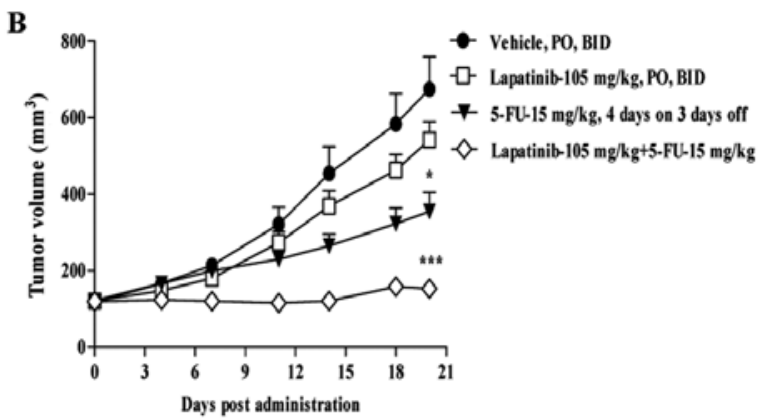

D

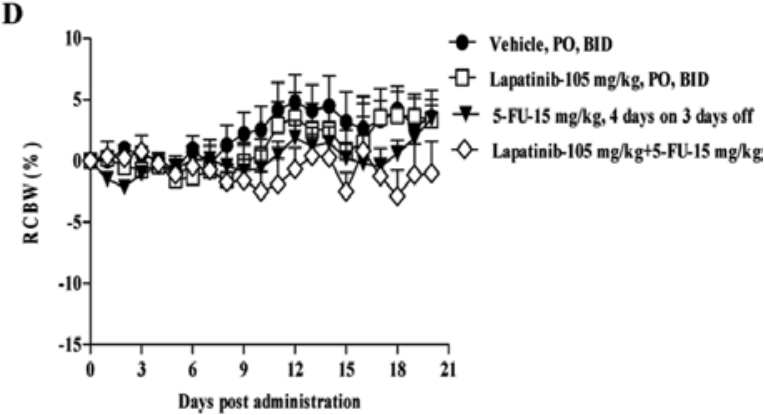

$\mathbf{F}$

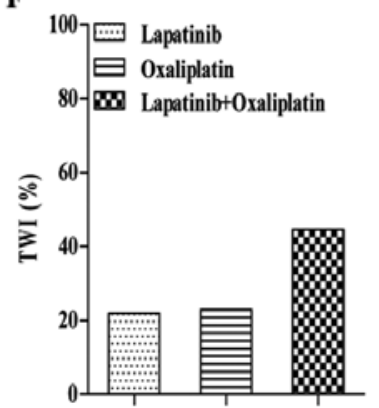

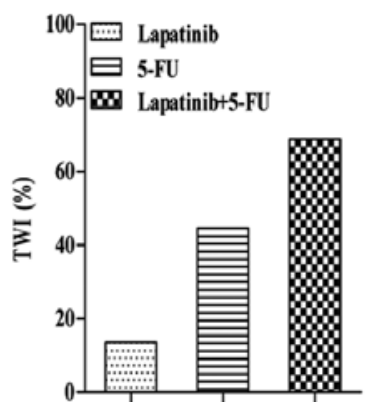

Figure 4. Antitumor activity of lapatinib, oxaliplatin, or 5-FU administered alone or in combination against primary ESCC models. (A) Tumor growth curves for ESCC primary models after treatment with lapatinib $(105 \mathrm{mg} / \mathrm{kg}$, twice daily), oxaliplatin $(6 \mathrm{mg} / \mathrm{kg}$, once weekly), or lapatinib in combination with oxaliplatin for 3 weeks. (B) Tumor growth curves for ESCC primary models after treatment with lapatinib (105 mg/kg, twice daily), 5-FU (15 mg/kg, 4 days per week), or lapatinib in combination with 5-FU. (C and D) Body weight was measured daily in each mouse to determine the relative change in body weight (RCBW). Error bars represent standard error of the mean. Repeated-measures analysis of variance showed statistically significant effects ("P $<0.05$ and $\left.{ }^{* * * *} \mathrm{P}<0.001\right)$ in both combination groups compared with the individual drugs. (E) Tumor growth inhibition and (F) tumor weight inhibition following treatment with lapatinib, oxaliplatin, or 5-FU alone or lapatinib in combination with either oxaliplatin or 5-FU.

5-FU induced a greatest decrease in the phosphorylation of EGFR and HER2 in primary ESCC models as compared with each drug alone or lapatinib in combination with oxaliplatin. The expression levels of pERK were slightly downregulated by lapatinib in combination with either oxaliplatin or 5-FU. There were no considerable increases in the blocking of pAKT expression in mice treated with lapatinib in combination with 5-FU, as compared with each drug alone or lapatinib in combination with oxaliplatin. These results suggest that, in primary ESCC models, blockade of pEGFR and pHER2 is beneficial for the synergistic inhibition of tumor growth following treatment with lapatinib in combination with 5-FU.

\section{Discussion}

Lapatinib is currently approved for the treatment of patients with HER2-positive metastatic breast cancer whose disease has progressed following trastuzumab-based therapy, and is licensed for use in these patients in combination with capecitabine (26). Based on these data and the increasing interest in the roles of EGFR and HER2 in gastric and esophageal cancer, we sought to evaluate the therapeutic potential of this dual TKI for the treatment of esophageal cancer. We also sought to compare the antitumor effects of lapatinib with standard chemotherapeutic drugs alone or in combination, and to understand the mechanism of action of these drugs.

In seven primary ESCC cells, the expression levels of EGFR and HER2 (total protein and phosphorylated protein) varied greatly. As previously reported in breast as well as in gastric cancer, there was no correlation between lapatinib activity and EGFR protein expression (27-29). Similarly, no correlation was found between lapatinib activity and HER2 protein expression. Several recent studies have examined the roles of lapatinib administered alone or in combination 
A

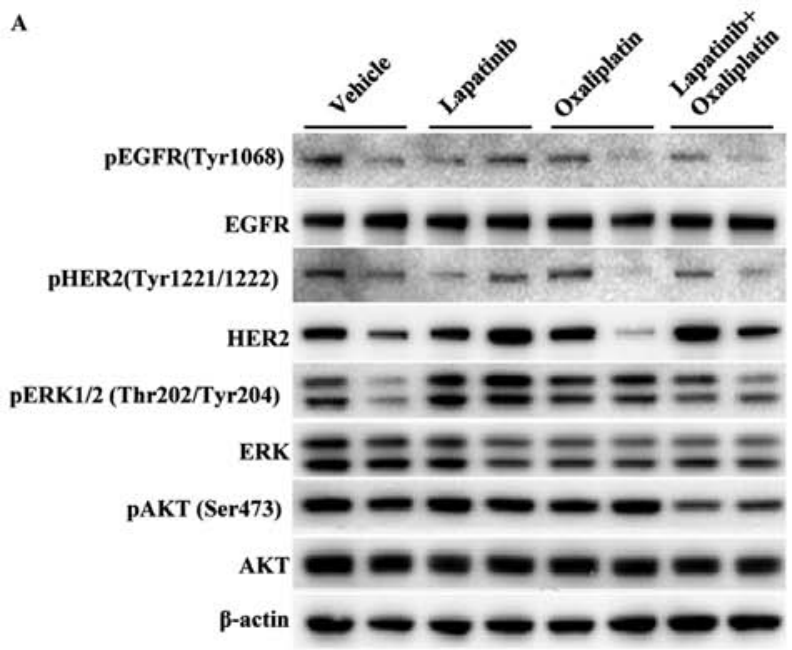

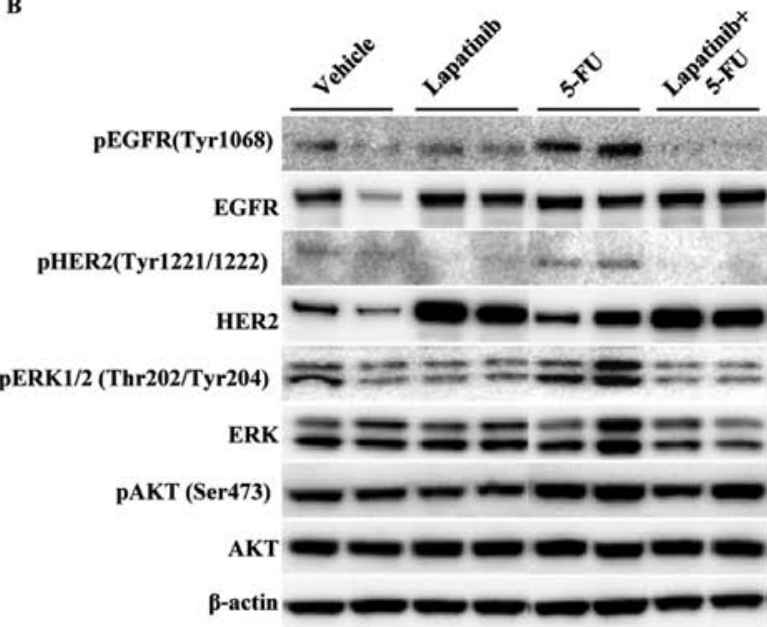

Figure 5. Effects of lapatinib, oxaliplatin, or 5-FU alone, or lapatinib in combination with either oxaliplatin or 5-FU on the expression levels of EGFR, HER2, and their downstream signaling components. pERK expression was slightly decreased in the lapatinib alone and lapatinib in combination with 5-FU groups. (A) No significant changes were seen for lapatinib in combination with oxaliplatin. (B) Lapatinib in combination with 5-FU suppressed EGFR and HER2 phosphorylation in the xenografts. Data represent one out of three independent experiments, each with comparable results.

with standard chemotherapies in esophageal cancer cell lines in vitro $(21,30)$. Our study provides further insight into the role of the HER family in esophageal cancer. First, our panel of primary tumor cells was directly derived from ESCC tissue samples obtained from Chinese patients. The physiologic condition differs greatly between primary tumor cells and long-established tumor cell lines. For example, primary tumor cells better reflect the in vivo situation (31-33). Our primary tumor cells were directly derived from ESCCs, and are therefore more clinically relevant than cell lines. Indeed, we found that the activity of ESCC tumor cells can be modulated by blocking the EGFR and HER2 signaling pathways.

The doses of lapatinib, oxaliplatin, and 5-FU used in the present study were determined based on the results of maximum tolerated dose studies performed in nude mice (data not shown). Efficacy studies in primary ESCC xenograft models showed that lapatinib $(105 \mathrm{mg} / \mathrm{kg})$ in combination with 5-FU induced near-complete tumor regression in all the mice. The effects of this combination were much greater than those achieved using either drug alone or with lapatinib combined with oxaliplatin. We also found that the synergistic antitumor effects of lapatinib in combination with 5-FU were probably mediated by changes in cell signaling. The levels of phosphorylated EGFR and HER2 were much lower following treatment with lapatinib in combination with 5-FU compared with either agent alone or with lapatinib in combination with oxaliplatin. Therefore, our data suggest that inhibition of the EGFR/HER2 signaling pathway by combining a chemotherapeutic drug and a TKI may augment the effects of both agents on the downstream signaling pathways. The synergy observed for this combination may have important clinical implications. For example, recent studies using breast cancer models have revealed that the accumulation of inactive HER2 receptor, as induced by lapatinib, enhances trastuzumab activity through antibody-dependent cellular cytotoxicity (34). Although our results are preliminary, they support the ongoing investigation of lapatinib in esophageal cancer as well as its possible combination with 5-FU in tumors overexpressing EGFR and
HER2. The results also suggest that the addition of anti-EGFR/ anti-HER2 therapy to standard chemotherapeutic drugs could have direct clinical benefits, and makes the investigation of additional anti-EGFR/anti-HER2 therapies in esophageal cancer particularly timely.

In the present study, we focused on the effects of lapatinib alone or in combination with standard chemotherapeutic drugs for esophageal cancer. Several clinical trials have suggested that the activity of anti-EGFR drugs seems to be limited to tumors of the gastroesophageal junction, with the response to both erlotinib and gefitinib being approximately $10 \%(35,36)$. In addition, in a clinical study of lapatinib in upper gastrointestinal cancer, disease control (prolonged stable disease) was only detected in patients with HER2-amplified disease. Our in vitro and in vivo observations support these clinical findings and the ongoing development of lapatinib in patients with tumors overexpressing HER2 (37,38).

Esophageal carcinoma is a highly malignant and prevalent cancer in China, for which the existing treatments have limited potential in reducing morbidity and mortality. Therefore, there is an urgent need to develop and refine new combinatorial therapies for this cancer. We have shown that lapatinib in combination with 5-FU has a significant synergistic therapeutic effect against ESCCs overexpressing EGFR and HER2. Lapatinib not only had a direct biological effect in terms of inhibiting the growth of ESCC primary cells in vitro, but also augmented the antitumor effects of 5-FU in primary ESCC models in vivo. Therefore, a regimen in which lapatinib is combined with an established chemotherapeutic drug represents a promising strategy for most patients with ESCC.

\section{Acknowledgements}

The present study was supported by grants from the One Hundred Talents Program of the Chinese Academy of Sciences, the National Natural Science Foundation (31070802), and the Ministry of Science and Technology of China (2009CB919000 and 2011BAK10B00). 


\section{References}

1. Demeester SR: Epidemiology and biology of esophageal cancer. Gastrointest Cancer Res 3: S2-S5, 2009.

2. Parkin DM, Bray F, Ferlay J and Pisani P: Global cancer statistics, 2002. CA Cancer J Clin 55: 74-108, 2005.

3. Blot W, McLaughlin J and Fraumeni J: Esophageal cancer. In: New Jersey Comprehensive Cancer Control Plan, 2008-2012. Schottenfeld D and Fraumeni J (eds). Oxford University Press, New York, pp697-706, 2006.

4. Ishikura S, Nihei K, Ohtsu A, et al: Long-term toxicity after definitive chemoradiotherapy for squamous cell carcinoma of the thoracic esophagus. J Clin Oncol 21: 2697-2702, 2003.

5. Tepper J, Krasna MJ, Niedzwiecki D, et al: Phase III trial of trimodality therapy with cisplatin, fluorouracil, radiotherapy, and surgery compared with surgery alone for esophageal cancer: CALGB 9781. J Clin Oncol 26: 1086-1092, 2008.

6. Del Campo JM, Hitt R, Sebastian P, et al: Effects of lapatinib monotherapy: results of a randomised phase II study in therapynaive patients with locally advanced squamous cell carcinoma of the head and neck. Br J Cancer 105: 618-627, 2011.

7. Scaltriti $\mathrm{M}$ and Baselga J: The epidermal growth factor receptor pathway: a model for targeted therapy. Clin Cancer Res 12 5268-5272, 2006.

8. Klapper LN, Kirschbaum MH, Sela M and Yarden Y: Biochemical and clinical implications of the ErbB/HER signaling network of growth factor receptors. Adv Cancer Res 77: 25-79, 2000.

9. Baselga J and Arteaga CL: Critical update and emerging trends in epidermal growth factor receptor targeting in cancer. J Clin Oncol 23: 2445-2459, 2005.

10. Roskoski R Jr: The ErbB/HER receptor protein-tyrosine kinases and cancer. Biochem Biophys Res Commun 319: 1-11, 2004.

11. Witton CJ, Reeves JR, Going JJ, Cooke TG and Bartlett JM: Expression of the HER1-4 family of receptor tyrosine kinases in breast cancer. J Pathol 200: 290-297, 2003.

12. Slamon DJ, Godolphin W, Jones LA, et al: Studies of the HER-2/ neu proto-oncogene in human breast and ovarian cancer. Science 244: 707-712, 1989.

13. Moasser MM: The oncogene HER2: its signaling and transforming functions and its role in human cancer pathogenesis. Oncogene 26: 6469-6487, 2007.

14. Hanawa M, Suzuki S, Dobashi Y, et al: EGFR protein overexpression and gene amplification in squamous cell carcinomas of the esophagus. Int J Cancer 118: 1173-1180, 2006.

15. Mimura K, Kono K, Hanawa M, et al: Frequencies of HER-2/neu expression and gene amplification in patients with oesophageal squamous cell carcinoma. Br J Cancer 92: 1253-1260, 2005.

16. Kawaguchi Y, Kono K, Mimura K, et al: Targeting EGFR and HER-2 with cetuximab- and trastuzumab-mediated immunotherapy in oesophageal squamous cell carcinoma. Br J Cancer 97: 494-501, 2007.

17. Higuchi K, Koizumi W, Tanabe S, et al: Current management of esophageal squamous-cell carcinoma in Japan and other countries. Gastrointest Cancer Res 3: 153-161, 2009.

18. Ilson DH: Esophageal cancer chemotherapy: recent advances. Gastrointest Cancer Res 2: 85-92, 2008.

19. Rusnak DW, Alligood KJ, Mullin RJ, et al: Assessment of epidermal growth factor receptor (EGFR, ErbB1) and HER2 (ErbB2) protein expression levels and response to lapatinib (Tykerb, GW572016) in an expanded panel of human normal and tumour cell lines. Cell Prolif 40: 580-594, 2007.

20. Xia W, Mullin RJ, Keith BR, et al: Anti-tumor activity of GW572016: a dual tyrosine kinase inhibitor blocks EGF activation of EGFR/erbB2 and downstream Erk1/2 and AKT pathways. Oncogene 21: 6255-6263, 2002.
21. Mimura K, Kono K, Maruyama T, et al: Lapatinib inhibits receptor phosphorylation and cell growth and enhances antibody-dependent cellular cytotoxicity of EGFR- and HER2overexpressing esophageal cancer cell lines. Int J Cancer 129: 2408-2416, 2011.

22. Kim HP, Yoon YK, Kim JW, et al: Lapatinib, a dual EGFR and HER 2 tyrosine kinase inhibitor, downregulates thymidylate synthase by inhibiting the nuclear translocation of EGFR and HER2. PLoS One 4: e5933, 2009.

23. Blackwell KL, Burstein HJ, Storniolo AM, et al: Randomized study of Lapatinib alone or in combination with trastuzumab in women with ErbB2-positive, trastuzumab-refractory metastatic breast cancer. J Clin Oncol 28: 1124-1130, 2010.

24. Cameron D, Casey M, Press M, et al: A phase III randomized comparison of lapatinib plus capecitabine versus capecitabine alone in women with advanced breast cancer that has progressed on trastuzumab: updated efficacy and biomarker analyses. Breast Cancer Res Treat 112: 533-543, 2008.

25. Chung KY, Shia J, Kemeny NE, et al: Cetuximab shows activity in colorectal cancer patients with tumors that do not express the epidermal growth factor receptor by immunohistochemistry. J Clin Oncol 23: 1803-1810, 2005.

26. Geyer CE, Forster J, Lindquist D, et al: Lapatinib plus capecitabine for HER2-positive advanced breast cancer. N Engl J Med 355: 2733-2743, 2006.

27. Wainberg ZA, Anghel A, Desai AJ, et al: Lapatinib, a dual EGFR and HER2 kinase inhibitor, selectively inhibits HER2-amplified human gastric cancer cells and is synergistic with trastuzumab in vitro and in vivo. Clin Cancer Res 16: 1509-1519, 2010.

28. Zhang D, Pal A, Bornmann WG, et al: Activity of lapatinib is independent of EGFR expression level in HER2-overexpressing breast cancer cells. Mol Cancer Ther 7: 1846-1850, 2008.

29. Konecny GE, Pegram MD, Venkatesan N, et al: Activity of the dual kinase inhibitor lapatinib (GW572016) against HER-2overexpressing and trastuzumab-treated breast cancer cells. Cancer Res 66: 1630-1639, 2006.

30. Guo XF, Zhu XF, Zhong GS, Deng BG, Gao ZT and Wang H: Lapatinib, a dual inhibitor of EGFR and HER2, has synergistic effects with 5-fluorouracil on esophageal carcinoma. Oncol Rep 27: 1639-1645, 2012.

31. Nolan GP: What's wrong with drug screening today. Nat Chem Biol 3: 187-191, 2007.

32. Kamb A: What's wrong with our cancer models? Nat Rev Drug Discov 4: 161-165, 2005

33. Masters JR: HeLa cells 50 years on: the good, the bad and the ugly. Nat Rev Cancer 2: 315-319, 2002.

34. Scaltriti M, Verma C, Guzman M, et al: Lapatinib, a HER2 tyrosine kinase inhibitor, induces stabilization and accumulation of HER 2 and potentiates trastuzumab-dependent cell cytotoxicity. Oncogene 28: 803-814, 2009.

35. Dragovich T, McCoy S, Fenoglio-Preiser CM, et al: Phase II trial of erlotinib in gastroesophageal junction and gastric adenocarcinomas: SWOG 0127. J Clin Oncol 24: 4922-4927, 2006.

36. Ferry DR, Anderson M, Beddard K, et al: A phase II study of gefitinib monotherapy in advanced esophageal adenocarcinoma: evidence of gene expression, cellular, and clinical response. Clin Cancer Res 13: 5869-5875, 2007.

37. Janmaat ML, Gallegos-Ruiz MI, Rodriguez JA, et al: Predictive factors for outcome in a phase II study of gefitinib in second-line treatment of advanced esophageal cancer patients. J Clin Oncol 24: 1612-1619, 2006.

38. Hecht J, Urba S and Koehler M et al: Lapatinib monotherapy in recurrent upper gastrointestinal malignancy: phase II efficacy and biomarker analyses. Proc GI Cancer Symposium, 2008. 